Herz 2011 · 36:71-72

DOI 10.1007/s00059-011-3435-6

(c) Urban \& Vogel 2011

\section{G. Hasenfuß ${ }^{1}$ - B. Maisch ${ }^{2}$}

${ }^{1}$ Universitätsmedizin, Abtl. Kardiologie und Pneumologie, Georg-August-Universität Göttingen, Göttingen

${ }^{2}$ Klinik für Innere Medizin - Kardiologie, Philipps-Universität Marburg, Marburg

\title{
Herzinsuffizienz - gezielte Diagnose unterschiedlicher Ursachen oder doch nur diagnostische und therapeutische Endstrecke?
}

Dass die Herzinsuffizienz keine umschriebene Krankheit ist, sondern ein Syndrom mit unterschiedlichen Ursachen, ist eine Binsenwahrheit und Lehrbuchwissen. Aus diesem Wissen folgt auch, dass neben der koronaren Herzerkrankung, die heute an jeder größeren, aber auch kleineren Klinik und in den Praxen niedergelassener Kardiologen erkannt und interventionell behandelt wird, auch die anderen Ursachen einer Herzschwäche erkannt und diagnostiziert werden sollten. Eine vereinfachte Einteilung der Herzinsuffizienz in eine ischämische und eine nichtischämische Kardiomyopathie ist ebenso unzulässig wie die Reduktion der Behandlung der koronaren Herzerkrankung auf die PTCA und/oder Stent-Implantation.

Herz hat sich in verschiedenen Themenheften in den letzten Jahren mit der Diagnostik und Therapie der unterschiedlichen Ursachen der Herzschwäche befasst und differenzierte Behandlungsempfehlungen zum Bestandteil seiner Übersichtshefte gemacht, damit neben den in den Leitlinien beschriebenen allgemeinen Prinzipien der Therapie des Terminalstadiums der chronischen Herzinsuffzienz die Behandlung der zugrunde liegenden Krankheit nicht vergessen wird. Diese Selbstverständlichkeit erschließt sich unter immer wieder neuen Blickwinkeln auch aus diesem Herz-Heft zur Frühjahrstagung 2011 der Deutschen Gesellschaft für Kardiologie (DGK).
Der Aspekt der akuten Herzinsuffizienz als „therapeutische Endstrecke“ verschiedener Ursachen gilt für die initiale Behandlung des kardiogenen Schocks zur Aufrechterhaltung der vitalen Organperfusion. Unter diesem Gesichtspunkt, aber auch unter Berücksichtigung von pathophysiologischen Mechanismen arbeiten M. Buerke, H. Lemm, S. Dietz, und K. Werdan den kardiogenen Schock auf. Aber auch hier ist neben der Umsetzung der allgemeinen Schocktherapie z. B. beim Herzinfarkt die umgehende Revaskularisation des verschlossenen oder hochgradig stenosierten Koronargefäßes prognoserelevant und weicht damit vom generalistischen Blick einer für alle Ursachen geltenden Behandlung ab.

V. Hombach, N. Merkle, V. Rasche, P. Bernhardt und W. Rottbauer sehen in ihrem Beitrag zur Rolle der Kardio-MRT in der Diagnose und Prognosebeurteilung von Patienten mit Herzinsuffizienz das Syndrom bereits differenzierter, auch wenn im ersten Ansatz nur die Unterscheidung der ischämischen von der nichtischämischen Herzinsuffizienz vorgenommen wird. Als ein wesentliches Korrelat der hämodynamischen Funktionseinschränkung bewerten sie den Nachweis des LGE („late gadolinium enhancement").

A. Rokita, C.-M. Sag und L.S. Maier steuern Prinzipien der terminalen Endstrecke des herzinsuffizienten Myokards bei: Veränderungen der elektromechanischen Kopplung bewirken auf Zelle- bene eine Kontraktionsinsuffizienz, die vor allem durch einen verminderten Kalzium- $\left(\mathrm{Ca}^{2+}-\right)$ Transienten und eine gestörte $\mathrm{Ca}^{2+}$-Speicherfähigkeit des sarkoplasmatischen Retikulums (SR) bedingt ist. Die $\mathrm{Ca}^{2+} /$ Calmodulin-abhängige Proteinkinase II $\delta$ trägt über einen verminderten SR-Ca ${ }^{2+}$-Gehalt sowie einen verminderten $\mathrm{Ca}^{2+}$-Transienten zur intrazellulären Akkumulation von $\mathrm{Ca}^{2+}$-Ionen und damit zur Entstehung der diastolischen Dysfunktion bei.

Die diastolische Dysfunktion ist auch das entscheidende initiale Merkmal der diabetischen Kardiomyopathie (DCM), die B. Maisch, P. Alter und S. Pankuweit in 4 Stadien oder Verlaufsformen einteilen:

- Stadium I (diastolische DCM): Hier führt die Hyperglykämie bei gleichzeitiger Insulinresistenz zu einer Hochregulation der Betaoxidation. Die intramyokardiale Akkumulation der freien Fettsäuren führt zu Lipotoxizität und zu einer gestörten Kalziumhomöostase. Ihre Bindung an interstitielles und perivaskuläres Kollagen führt zu „advanced glycation endproducts" (AGE) und damit zu vermehrter Steifigkeit, Hypertrophie (auch ohne Hypertonie) und additiver Fibrose.

- Stadium II (diastolische und systolische DCM): Hier kommt es zur systolischen und diastolischen Funktionsstörung. Erst in den späteren beiden Stadien kommt die Beteili- 
gung der kleinen Gefäße bzw. der epikardialen Koronarien prognoserelevant hinzu.

- Stadium III (Mikrovaskulopathie zusätzlich zu Stadium I und II).

- Stadium IV (Makroangiopathie mit Ischämie oder Infarzierung zusätzlich zu den Stadien I bis III).

Die Verbreitung des transkutanen kathetergestützten Aortenklappenersatzes (TAVI) und ebenso der herzchirurgisch geführten transapikalen Klappenimplantation hat in der letzten Zeit sprunghaft zugenommen. Beide Eingriffe zielen auf Patienten, die nicht für Operationen am offenen Herzen geeignet sind. Sie bieten insbesondere älteren und alten Patienten Chancen zur Verbesserung der Lebensqualität und ggf. auch der individuelle Prognose bei einer Krankheit, die innerhalb von 6 bis 12 Monaten bei rund 50\% der Patienten unbehandelt zum Tode führt. H.R. Figulla beschäftigt sich in seinem Beitrag mit der Frage, ob die TAVI, die eine spezifische Therapie der Herzinsuffizienz bei Aortenstenose darstellt, bereits wirklich im Sinne der evidenzbasierten Medizin etabliert ist.

C. Nesselmann, A. Kaminski und C. Steinhoff fassen in ihrem Beitrag die wesentlichen aktuellen registrierten Studien zur kardialen Stammzelltransplantation zusammen. Der Stammzelltransplantation sowie den mit ihr verbundenen Hoffnungen und bis heute unerfüllten Erwartungen hat Herz im Jahr 2010 zwei Hefte gewidmet. Die Autoren stellen auch vorläufige Daten einer Anwendungsbeobachtung eines zusätzlichen intramyokardialen Einsatzes von CD133+-Stammzellen bei 10 Patienten vor, die einer Mitralklappenoperation oder -raffung und einer Stammzelltransplantation unterzogen werden. Da alle Patienten neben der Mitralklappenoperation auch Stammzellen erhielten, lässt sich der prognostische Wert dieser Maßnahme ohne Kontrollgruppe leider nicht abschätzen.

Psychische und soziale Faktoren interagieren mit der Herzinsuffizienz in vielfältiger Weise. In seinem Beitrag weist $C$. Herrmann-Lingen darauf hin, dass Depression und psychischer Distress über eine Maladaptation psychophysiologi- scher Prozesse an der Entstehung und Progredienz der Herzinsuffizienz beteiligt sein können. Herzinsuffizienzbedingte Stressoren können über die Aktivierung inflammatorischer Prozesse zu Erschöpfungsgefühlen und depressiven Störungen beitragen. Hieraus kann ein Circulus vitiosus entstehen, der nicht nur die Lebensqualität beeinträchtigt, sondern auch prognosebestimmend sein kann.

Dieses Herz-Heft zur Frühjahrstagung der DGK bietet jedem Kardiologen etwas für den klinischen, aber auch den wissenschaftlichen Alltag, ohne deshalb eine Art Patchwork der Herzinsuffizienz zu sein. Es beleuchtet Diagnostik und Therapie des Syndroms nicht nur in der terminalen Phase einer schlussendlich infausten Erkrankung, sondern widmet sich auch der Pathogenese und zeigt aktuelle diagnostische und therapeutische Optionen zum Nutzen der Patienten mit Herzinsuffizienz auf.

Herzlich willkommen zur Frühjahrstagung der DGK, die sich in ihren Hauptsitzungen diesen Themen besonders verschrieben hat!

Gerd Hasenfuß -

Präsident der Frühjahrstagung 2011

Bernhard Maisch - Herausgeber Herz

\section{Korrespondenzadresse}

\section{Prof. Dr. B. Maisch}

Klinik für Innere Medizin - Kardiologie,

Philipps-Universität Marburg

Baldingerstraße, 35043 Marburg

maisch@mailer.uni-marburg.de 